Key-words: Aquatic environment. Pesticides. Vector control. Africa.

\title{
IMPACT DE LA LUTTE ANTIVECTORIELLE SUR L'ENVIRONNEMENT AQUATIQUE
}

\author{
C. LÉVÊQUE
}

RÉSUMÉ

L'impact sur l'environnement aquatique des traitements chimiques antivectoriels contre l'Onchocercose, la Trypanosomiase, le Paludisme et la Bilharziose, dépend des pesticides utilisés et des stratégies adoptées. A partir de l'expérience du Programme de lutte contre l'Onchocercose en Afrique de l'Ouest, nous présen- tons dans quel contexte et en fonction de quels objectifs, il est possible de mettre en place un programme de surveillance de l'environnement afin de minimiser l'impact des traitements. En Afrique tropicale, la pression des pesticides chimiques antivectoriels, s'est réduite dans les années 1980 .

SUMmary: Vector control: its impact on the aquatic environment.

Environmental impact of pesticides used in vector control programmes against Onchocerciasis, Trypanosomiasis, Malaria and Bilharziasa, depends on chemicals used and control strategies. Dealing with the experience of the Onchocerciasis control Programme in West Africa, we introduce in what perspective, it is possible to establish an environmental monitoring programme in order to minimize the impact of treatments. In tropical Africa, vector control chemicals pressure, decreased in the years 1980 .
Il n'est guère possible en quelques pages de présenter, même sommairement, l'ensemble des résultats concernant l'impact de la lutte antivectorielle sur l'environnement aquatique. Divers travaux récents (Dejoux, 1988; Everts et Koeman, 1987; Koeman et al., 1980; Lévêque et al., 1988; Yaméogo et al., 1989) font la synthèse des résultats obtenus dans différents programmes, et le lecteur intéressé pourra s'y reporter avec profit. Nous nous proposons donc ici de situer rapidement le contexte dans lequel s'effectuent ces études d'impact, afin d'essayer de dégager une démarche et des principes généraux en vue d'assurer une bonne surveillance écologique des milieux aquatiques soumis à des épandages de pesticides. Nous nous sommes beaucoup appuyés pour cela sur l'expérience acquise par le Programme de lutte contre l'Onchocercose en Afrique de l'Ouest, qui, en raison de la surface traitée (plus d'un million de kilomètres carrés), de sa durée (il a débuté en 1973) et des quantités importantes d'insecticides utilisées, s'est doté dès son origine d'un programme de surveillance de l'environnement aquatique particulièrement actif, et pour le moins unique en son genre pour des programmes de lutte de cette envergure.

En milieu tropical, beaucoup de maladies parasitaires sont liées à la présence de l'eau qui est indispensable au bon déroulement du cycle biologique du vecteur :

Directeur de Recherches, ORSTOM, 213, rue Lafayette, F 75480 Paris Cedex 10, France.
- moustiques (Anophèles et Aedes) transmettant le paludisme et la filariose de Bancroft, ou simulies transmettant l'onchocercose, dont les stades préimaginaux sont aquatiques, alors que les adultes sont aériens,

- mollusques gastéropodes transmettant la bilharziose ou copépodes planctoniques transmettant la dracunculose, dont le développement est entièrement aquatique,

- glossines (ou tsé-tsé) transmettant la trypanosomiase, dont les aires de repos des adultes sont les galeries forestières bordant les cours d'eau.

Si des moyens mécaniques sont parfois utilisables pour lutter contre certaines parasitoses, dans beaucoup de cas, le contrôle des vecteurs par l'utilisation de pesticides est le seul moyen d'interrompre la transmission, en l'absence de médicaments appropriés à une chimiothérapie de masse. Par le passé, cette lutte chimique contre le vecteur a pu apparaître quelque peu anarchique, et des impacts importants ont été signalés. Cette situation qui était considérée par certains comme le prix à payer pour éradiquer les maladies parasitaires, a fort heureusement évolué depuis et les grands programmes menés actuellement, tout en se préoccupant de contrôler l'espèce-cible pour limiter la transmission, s'efforcent de plus en plus d'utiliser des produits les moins toxiques possible pour l'environnement aquatique. Cette évolution est la conséquence de l'action conjuguée de divers facteurs. En premier lieu, une prise de conscience sur le plan international, des risques liés à une dégradation de l'environnement. Mais surtout, la mise en évidence à la fin des années 1960 d'une grave contamination de 
l'homme par le DDT, en raison de son accumulation dans la chaîne trophique, a fortement alarmé les pays industrialisés qui ont interdit l'usage de ce pesticide. Depuis cette époque, l'utilisation, l'impact et le devenir des insecticides dans l'environnement sont beaucoup mieux contrôlés, y compris dans les pays tropicaux.

\section{1 - LES PESTICIDES UTILISÉS}

L'usage des pesticides pour lutter contre les vecteurs, s'est généralisé après la seconde guerre mondiale, avec une utilisation massive d'organochlorés. Depuis, de nombreuses autres molécules ont été commercialisées, avec des succès divers. Compte tenu des phénomènes de résistance pouvant apparaître chez les espèces vectrices, la recherche de nouveaux composés se poursuit activement, encouragée par les utilisateurs soucieux de posséder les produits de remplacement, mais également par les écologistes soucieux de voir sélectionner des molécules peu toxiques pour l'environnement. Si des milliers de formulations sont théoriquement disponibles, seules quelques-unes sont réellement utilisées à grande échelle dans la lutte contre les vecteurs.

Les organochlorés sont, en général, peu solubles dans l'eau mais fortement liposolubles. Ils se dégradent lentement dans l'eau (50\% du DDT seulement disparaît entre 7 et 10 ans) et s'accumulent dans les sédiments et les chaînes trophiques qu'ils contaminent. En raison de cette rémanence et de leur toxicité, les organochlorés sont de moins en moins utilisés pour les traitements en milieu aquatique.

Les organophosphorés ont une durée de vie beaucoup plus courte (quelques semaines). Le téméphos, en particulier, est rapidement métabolisé par les organismes et se dégrade assez rapidement en milieu naturel. C'est dans ce groupe que l'on trouve, en général, les composés chimiques les moins toxiques pour la faune aquatique non cible. Ces composés ont un effet sur l'activité cholinestérase des poissons (Dejoux, 1988).

Pami les carbamates, le carbosulfan est actuellement utilisé dans la lutte antisimulidienne. A condition d'être utilisé en période de crue, et pour un nombre limité de cycles de traitement, ce pesticide a un impact acceptable sur la faune non cible.

Les pyréthinoïdes sont, en général, toxiques et nécessitent d'être utilisés avec précaution. Ils sont particulièrement dangereux pour les crustacés planctoniques et les crevettes (Everts et Koeman, 1988), mais se dégradent rapidement en milieu naturel.

Le Bacillus thuringiensis israelensis, insecticide d'origine biologique, est en réalité une protéine cristallisée toxique produite par les spores d'une bactérie. Très sélectif, cet insecticide est assez peu toxique pour la faune non cible, et peut être utilisé en particulier pour les faibles débits et dans les eaux stagnantes.

Des pesticides d'autres familles chimiques sont également utilisés dans la lutte contre les mollusques: Niclosamide (Bayluscide) et N-tritylmorpholine (Frescon).

\section{2 - LES MÉTHOdES DE LUTTE CHIMIQUE}

Tout programme antivectoriel présente sa spécificité qui dépend de la biologie et de l'écologie des vecteurs, ainsi que de la zone géographique couverte. Schématiquement cependant, on peut distinguer des caractéristiques propres à chacune des grandes endémies :

- Onchocercose : la lutte porte sur les stades larvaires habitant les zones de rapides des rivières. Il faut donc repérer ces gîtes pour les traiter ensuite. La durée de développement larvaire très courte ( 8 à 10 jours) de Simulium damnosum s. $l$. implique des traitements fréquents (hebdomadaires), alors que la durée de vie du ver adulte est de 12 à 15 ans chez l'homme. Pour espérer interrompre la transmission, il faut donc traiter au minimum pendant 15 ans. Les épandages se font directement dans la rivière, en amont des gîtes larvaires. Les insecticides les plus utilisés par le Programme de lutte contre l'Onchocercose en Afrique de l'Ouest, selon un système de rotation au cours de l'année, sont le Bt H 14 et, éventuellement, le téméphos en saison sèche, le chlorphoxime, la perméthrine et le carbosulfan en raison des pluies (Lévêque et al., 1989). Des essais de lutte contre les adultes n'ont pas donné de résultats probants.

- Trypanosomiase : la lutte concerne les glossines adultes dont les aires de repos sont limitées aux forêts galeries le long des fleuves en saison sèche. Les épandages réalisés par voie aérienne dans ces boisements riverains, suscitent très souvent une retombée directe d'insecticide dans l'eau, malgré les précautions qui peuvent être prises. Deux stratégies sont utilisées pour le contrôle des vecteurs : des traitements utilisant des produits rémanents appliqués à concentration élevée, et nécessitant seulement quelques épandages chaque année; des traitements utilisant des produits peu rémanents à faible dose, mais nécessitant des épandages plus fréquents (4 à 5 sur 2 à 3 mois). Les insecticides les plus fréquemment utilisés sont des organochlorés (DDT auparavant, dieldrine, endosulfan) et des pyréthrinoïdes (perméthrine, deltaméthrine) (Koeman et al., 1980; Dejoux, 1988).

- Paludisme : malgré l'existence d'une chimiothérapie de masse, la démoustification est un moyen de lutte non négligeable. Les adultes peuvent être attaqués par pulvérisation aérienne sur leurs lieux de repos, essentiellement en agglomération, et les larves par application d'insecticides dans les milieux aquatiques où elles se développent, qui sont très généralement des milieux stagnants où les insecticides rémanents auront un impact durable sur la faune aquatique. Divers organophosphorés ont été utilisés.

- Bilharziose : les mollusques vecteurs (Bullinus et Biom- 
phalaria) peuplent les plans d'eau stagnante ou semistagnante, les milieux artificiels et les réseaux d'irrigation qui constituent des milieux favorables à leur développement. Pendant très longtemps, les molluscicides utilisés ont été des produits très toxiques provoquant de grandes mortalités de poissons (Bayluscide et Frescon). Les campagnes d'éradication n'ayant pas toujours donné de résultats très concluants et coûtant fort cher (Brown, 1980), ces produits sont actuellement moins utilisés. La nécessité de rechercher de nouveaux produits de synthèse, efficaces et peu coûteux, a été souvent rappelée (Anonyme, 1985). On fonde actuellement quelques espoirs dans des molluscicides d'origine végétale. Cependant, il conviendrait de faire des essais plus nombreux sur le terrain pour tester leur efficacité et surtout leur impact sur l'environnement, car aucune étude écotoxicologique à long terme ne semble avoir été consacrée à ces produits.

\section{3 - SÉLECTION DES PESTICIDES}

L'apparition de résistances chez les vecteurs, et la nécessité de trouver des pesticides de remplacement, ainsi que la mise à disposition sur le marché de nouveaux produits, sont autant de raisons pour un Programme de lutte contre les vecteurs de rechercher en permanence des pesticides opérationnels. Les critères de sélection sont généralement les suivants :

- être totalement efficace contre la cible, rieurs,

- être d'un prix attractif et d'emploi aisé dans les conditions opérationnelles.

Par rapport à l'environnement aquatique, d'autres exigences peuvent être formulées :

- ne pas utiliser de pesticides donnant des produits de dégradation toxiques et rémanents s'accumulant dans les chaînes trophiques,

- ne pas avoir d'impact sur les poissons à différentes étapes de leur cycle biologique,

- ne pas provoquer à long terme, un déséquilibre des écosystèmes dans les conditions normales d'application. Cela implique que l'abondance relative des espèces non cibles ne doit pas subir de modifications majeures et irréversibles, et que l'insecticide ne provoque pas au niveau régional l'éradication définitive d'espèces aquatiques non cibles.

\section{4 - ÉVALUATION DE L'IMPACT EN MILIEU NATUREL}

L'impact sur l'environnement peut être évalué par une surveillance chimique et/ou biologique, les deux approches étant, bien entendu, complémentaires.

La surveillance chimique consiste à rechercher les résidus de pesticides dans l'eau, les sédiments et les tissus d'organismes vivants. Cependant, des problèmes d'ordre méthodologique liés à l'échantillonnage (Koeman et al., 1980), ainsi qu'à l'analyse des échantillons, ont fortement limité l'utilisation de ces techniques en milieu tropical.

La surveillance biologique consiste à suivre l'évolution temporelle des écosystèmes traités et de certaines de leurs composantes biologiques : diversité spécifique, composition et abondance des communautés, paramètres physiologiques (reproduction, activité enzymatique), etc. Comme il est impossible d'examiner tous les éléments, les recherches sont généralement concentrées sur deux ou trois groupes d'organismes :

- les poissons dont on peut penser que l'état des populations est le résultat des conditions générales de l'environnement dans lequel ils vivent (Munkittrick et Dixon, 1989). En raison de leur position en haut de la chaîne trophique, ils peuvent concentrer les résidus et être sensibles à la disparition de certaines catégories de proies. Mais la structure des populations peut également être modifiée par une mortalité accrue des adultes, un taux de survie plus faible des juvéniles ou une diminution de la fécondité. Il convient de rappeler également l'intérêt économique de ce groupe dans beaucoup de régions tropicales:

- les macrocrustacés qui constituent, dans certains milieux, une part importante de la biomasse et sont particulièrement sensibles à certaines familles d'insecticides;

- les invertébrés benthiques (larves d'insectes) en rivière, ou planctoniques en milieu stagnant, qui ont, au niveau de la surveillance, l'avantage d'être plus sensibles que les précédents et de pouvoir être facilement utilisés dans des tests de sensibilité. Ces organismes sont également les proies de beaucoup d'espèces de poissons.

L'homologation d'un pesticide pour des campagnes d'éradication en milieu naturel devrait comporter un certain nombre d'étapes permettant d'évaluer son impact sur la faune aquatique :

\section{Toxicité aiguë}

La plupart des produits utilisés en lutte antivectorielle ont fait l'objet de tests de toxicité au laboratoire. Menés en conditions contrôlées, ceux-ci ont pour but de déterminer les doses létales (LC50) pour un temps d'exposition donné au pesticide. Différents organismes, surtout de milieux tempérés, ont été testés : crustacés planctoniques (Daphnia), macrocrustacés, larves d'insectes aquatiques, poissons, etc. Il existe dans ce domaine une abondante littérature que nous ne développerons pas (voir Dejoux, 1988, pour une revue partielle). L'intérêt immédiat de ces tests de toxicité aiguë est de pouvoir comparer divers pesticides dans des conditions plus ou moins standardisées.

Les tests réalisés en milieu confiné ne sont pas toujours directement extrapolables au milieu tropical naturel. C'est 
pourquoi une méthode originale, celle des gouttières multiples (Troubat, 1981) a été développée en Afrique pour tester la toxicité directe des insecticides sur les invertébrés en eau courante et en condition semi-naturelle. Composé de plusieurs petites gouttières parallèles, l'appareil est, en partie, immergé in situ sur les gîtes à Simulium damnosum. Avec un tel système, on peut comparer simultanément l'impact de diverses concentrations d'un insecticide, ou l'impact de divers insecticides. Cette méthode est actuellement largement employée par le Programme de lutte contre l'Onchocercose. Si les résultats obtenus sont peut-être moins reproductibles, car obtenus dans des conditions non standardisées, ils ont cependant l'avantage d'être plus proches des conditions naturelles, et de concerner la faune autochtone.

\section{Impact à court terme}

Une fois l'insecticide retenu comme candidat potentiel pour les campagnes antivectorielles, il doit être testé dans les conditions opérationnelles. Un traitement réalisé sur une portion de rivière, par exemple, permet d'apprécier l'impact immédiat du produit sur la faune aquatique dans les conditions naturelles, en utilisant différentes techniques hydrobiologiques (Lévêque et al., 1979) : cycle de dérive des invertébrés et des poissons avant et après épandage, prélèvements au Surber, recherche visuelle d'organismes morts, etc.

\section{Impact à moyen terme}

L'impact d'un insecticide peut être plus ou moins important sur la faune non cible en fonction de la saison. L'effet de la température sur la toxicité de certains produits est bien connu, mais les conditions d'utilisation en fonction du cycle hydrologique sont également à prendre en compte, notamment en saison sèche quand les milieux aquatiques sont très réduits en zone intertropicale. Il est donc nécessaire d'évaluer l'impact au cours d'un ou deux cycles saisonniers, en effectuant, par exemple, une surveillance régulière de l'évolution de la faune aquatique dans quelques stations de référence, au moyen de techniques standardisées : filets à dérive pour les invertébrés et les poissons, par exemple, batterie de filets maillants pour les poissons, etc. (Lévêque et al., 1979).

\section{Impact à long terme}

Il est possible qu'un pesticide, lorsqu'il est utilisé régulièrement pendant plusieurs années pour la lutte antivectorelle, puisse avoir un effet limité ou difficilement décelable à court et moyen terme, mais un impact non négligable à long terme. Ces effets différés pourraient, par exemple, être une modification du potentiel reproducteur ou du taux de survie des juvéniles de poissons, la disparition d'espèces à long cycle de vie soumises à des traitements répétés, etc. Cette évaluation à long terme est réalisée dans les mêmes conditions que la précédente. Elle se justifie d'autant plus dans certaines stations de référence, que des insecticides différents sont utilisés en rotation tout au long de la campagne de traitement, afin d'éviter le développement de résistances chez le vecteur.

L'interprétation des résultats obtenus dans le cadre de ces différentes activités de surveillance n'est pas toujours sans poser de problèmes. Tout d'abord, l'extrapolation des résultats obtenus dans un contexte écologique donné (température, chimie des eaux, turbidité, morphologie des écosystèmes) ne doit se faire qu'avec prudence sachant que les effets d'un produit peuvent être variables selon les conditions d'utilisation. D'autre part, il n'est pas toujours aisé de faire la part respective des facteurs naturels et des traitements, dans l'évolution à long terme des systèmes. L'existence de stations témoins non traitées, permet néanmoins d'apprécier l'impact quand il existe. Enfin, l'effet d'un pesticide sera d'autant plus faible sur le long terme que les possibilités de recolonisation des zones traitées à partir de zones non traitées, seront importantes. Cette notion de zone refuge pour les organismes est fondamentale dans l'interprétation des effets potentiels. Un accident toxique aigu aura d'autant plus d'impact que les possibilités de recolonisation du milieu seront faibles. Ces potentialités de recolonisation sont, bien entendu, liées à la mobilité des organismes, mais également à la durée de leur cycle de développement.

\section{5 - ORGANISER LA SURVEILLANCE DE L'ENVIRONNE- MENT AQUATIQUE}

Pour évaluer scientifiquement et objectivement l'impact des traitements antivectoriels sur la faune aquatique, il est nécessaire de mettre en place un Programme de surveillance de l'environnement aquatique fonctionnant parallèlement au programme de lutte. Il y a peu d'exemples au monde d'une telle organisation, en dehors de celle mise en place par le Programme de lutte contre l'Onchocercose en Afrique de l'Ouest (Lévêque et al., 1989; Yaméogo et al., 1989). Dans ce programme, des traitements aériens antisimulidiens sont effectués chaque semaine sur potentiellement $30000 \mathrm{~km}$ de rivières. Conscients des risques encourus par l'environnement du fait de ces épandages qui doivent se poursuivre sur 15 à 20 ans, les pays donateurs ont accepté de financer le Programme de lutte, à la condition qu'une structure soit mise en place pour surveiller l'impact sur la faune non cible et le devenir des insecticides utilisés. Actuellement l'unité responsable des traitements comprend une équipe chargée de rechercher de nouveaux insecticides et de les tester sur les Simulies et la faune non cible et une équipe d'hydrobiologistes qui a pour tâches principales de réaliser un suivi plus approfondi des insecticides opérationnels ou potentiellement utilisables, et de coordonner l'activité d'équipes nationales chargées de suivre 
l'évolution à long terme de la faune aquatique dans des stations de surveillance sélectionnées, selon un protocole standardisé (Lévêque et al., 1979). Ces différentes équipes, assistées lorsque cela est nécessaire par des consultants extérieurs, rendent compte à un Groupe Écologique constitué de 5 experts indépendants, qui se réunit chaque année. Ce Groupe Écologique examine les résultats obtenus par les équipes d'hydrobiologistes, demande éventuellement des études complémentaires, et fait part de ses conclusions à un Comité d'Expert qui conseille le Programme, ainsi qu'au groupe des donateurs lors des réunions annuelles. Une telle structure a montré depuis plusieurs années son efficacité et a contribué à la crédibilité du Programme sur le plan de la protection de l'environnement aquatique.

\section{6 - CONCLUSIONS ET PESPECTIVES}

Les campagnes de lutte chimique contre les vecteurs menées en Afrique tropicale, notamment, ont connu une certaine vogue au cours des années 1960-1970, mais se sont un peu ralenties depuis. Les raisons en sont nombreuses et le souci de protéger l'environnement n'est pas toujours la préoccupation principale. Ainsi les conséquences écotoxicologiques de l'utilisation de certains organochlorés, l'apparition d'une résistance aux insecticides chez certains vecteurs, le succès relatif et le coût élevé d'un certain nombre de campagnes, ont modéré un peu les enthousiasmes des partisans de la lutte chimique. Dans certains cas, l'apparition de médicaments (bilharziose) ou les perspectives d'utilisation de moyens individuels (cas des moustiquaires imprégnées d'insecticides rémanents pour lutter contre le paludisme (Rozendahl, 1989); cas des pièges à glossines installés près des villages), ont également contribué à réduire la pression des pesticides. En réalité, on s'oriente de plus en plus vers des luttes antivectorielles intégrées, associant l'éducation sanitaire, médicaments, traitements chimiques, biologiques ou mécaniques, manipulation du milieu, etc. La lutte biologique a été prônée depuis longtemps contre les vecteurs de la bilharziose. On s'est intéressé à des espèces de mollusques compétitrices du vecteur, à des prédateurs (poissons en particulier), à des bactéries pathogènes et à des parasites. Mais, jusqu'à présent, ces méthodes n'ont eu qu'un succès limité et ne peuvent être envisagées à brève échéance comme des solutions alternatives pour une lutte à grande échelle. De même, si la contamination au laboratoire de larves de Culicides avec des Nématodes (Mermithidae) a donné de bons résultats, celle de larves de Simulies s'avère difficile in situ. La lutte mécanique est également employée pour éliminer des gîtes de moustiques en milieu urbain, pour assécher des régions marécageuses et lutter contre les moustiques ou mollusques vecteurs. Une manipulation des niveaux d'eau dans les réservoirs et canaux d'irrigation a souvent été recommandée.
Le seul Programme actuel de grande envergure utilisant massivement des insecticides est le Programme de lutte contre l'Onchocercose en Afrique de l'Ouest. Nous y avons fait largement référence dans ce travail, et l'on peut résumer la situation actuelle en disant que ce programme est un succès reconnu sur le plan de la lutte contre la maladie puisque celle-ci n'est plus endémique des zones traitées depuis 15 ans. En ce qui concerne l'impact sur l'environnement, tous les résultats obtenus montrent que cet impact n'est pas décelable à court, moyen et long terme sur les populations de poissons (Lévêque et al., 1988). Il a cependant été montré sur les invertébrés que chaque insecticide avait sa propre signature et affectait à des degrés divers les communautés naturelles. Cependant, aucune évolution irréversible n'a encore été constatée, et ici également, les résultats obtenus dans le cadre de la surveillance des milieux aquatiques, tendent à montrer que l'impact est modéré et que les systèmes ont conservé de grandes facultés de récupération une fois les traitements suspendus (Yaméogo et al., 1988).

En réalité, une meilleure connaissance du terrain et de la dynamique des populations du vecteur, ainsi qu'une meilleure maîtrise technique dans l'utilisation des insecticides, ont également permis d'alléger considérablement l'impact des traitements. Ce Programme, qui a associé depuis ses débuts une composante environnementale dans ses objectifs, a démontré la faisabilité d'une lutte chimique longue et efficace, sauvegardant pour l'essentiel l'environnement aquatique.

\section{RÉFÉRENCES}

Anonyme : Lutte contre la Schistosomiase. Rapport d'un Comité OMS d'experts. Rapport technique 728, OMS, 1985, 128 p.

Brown D. : Freshwater snails of Africa and their medical importance. Taylor and Fancis, London, 1980.

Dejoux C. : La pollution des eaux continentales africaines. Travaux et Documents, ORSTOM, Paris, 1988, n² 213.

Everts J. W., Koeman J. H. : The ecological impact of insecticides in connection to the control of tsetse flies in Africa: a review. In: Cavalloro, ed., Proc. of the CEC Int. Symp., Ispra, 4-6 March 1986. A. A. Balkema, Rotterdam, 1988.

Koeman J. H., Balk F., Takken W. : The environmental impact of tsetse control operations: a report of present knowledge. FAO Animal Production and health paper 7, Rev. 1, 1980, 71 p.

Kurtak D., Jamnbazck H., Meye R., Ocran M., Renaud P. : Evaluation of larvicides for the Control of Simulium damnosum s. $l$. (Diptères, Simulidae) in West Africa. J. Am. Mosquito Contr. Ass., 1987, 3, 201-210

Lévêque C. : The use of insecticides in the Onchocerciasis Control Programme and Aquatic Monitoring in West Africa. In: Bourdeau et al. (ed). Ecotoxicology and climate, SCOPE 38. J. Wiley and Sons, Chichester, 1989, 317-335.

Lévêque C., Fairhurst C. P., Abban K., Paugy D., Curtis M. S., Traore K. : Onchocerciasis Programme in est Africa. Ten years monitoring of fish populations. Chemosphere, 1988, 17, 421-440. 


\section{LÉVÊQUE}

Lévêque C., Odei M., Pugh-Thomas M. : The Onchocerciasi Control Programme and the monitoring of its effects on the riverine biology of the Volta River basin. In: F. H. Perring, K. Mellanby (eds), Ecological effects of pesticides. Linnean society Symposium seres 5. Academic Press, London, 1979.

Muirhead-Thompson R. C. : Pesticides and freshwater fauna. Academic Press, London, 1971, 248 p.

Munkittrick K. R., Dixon D. G. : A holistic approach to ecosystem health assessment using fish population characteristics. Hydrobiologia, 1989, 188, 123-135.
Rozendaal J. A. : Impregnated mosquito nets and curtains for self-protection and vector control? Trop. Dis. Bull., 1989, 86. Troubat J. J. : Dispositif à gouttières multiples destiné à tester in situ la toxicité des insecticides vis-à-vis des invertébrés benthiques. Rev. Hydrobiol. Trop., 1981, 14, 149-152.

Yaméogo L., Lévêque C., Traore K., Fairhurst C. P. : Dix ans de surveillance de la faune aquatique des rivières d'Afrique de l'Ouest traitées contre les Simulies (Diptera; Simulidae), agents vecteurs de l'Onchocercose humaine. Naturaliste Canad. Rev. Écol. Syst., 1988, 115, 287-298. 